BULLETIN (New Series) OF THE

AMERICAN MATHEMATICAL SOCIETY

Volume 37, Number 1, Pages 65-74

S 0273-0979(99)00800-9

Article electronically published on December 21, 1999

\title{
A MATHEMATICAL CRITIQUE OF SOME PHYSICAL THEORIES
}

\author{
G. D. BIRKHOFF
}

\section{Introduction}

My purpose today is to review some of the mathematical-physical theories of the past and of the present, indicating briefly the nature of certain concepts upon which these theories rest as well as the attendant logical difficulties, and even proposing modifications which have occurred to me. Of course the subject is too large for an address of this kind, but interest in mathematical-physical ideas is very widespread, and their importance for both mathematicians and physicists is profound. I feel more justified in choosing this subject because it has occupied so much of my attention recently.

\section{Geometry}

It goes without saying that geometry is the first and simplest of such theories, unless arithmetic be regarded as of this type. But the only assumption of a physical type underlying arithmetic is that material objects possess a permanent identity; and this can hardly be considered as an assumption if we are to do any thinking at all.

Now ordinary three-dimensional geometry of space is extremely simple in essence. Some day, when the field of knowledge has extended so far that simplification becomes necessary, ordinary geometry may be approached somewhat as follows:

(1) Geometry treats of elements called points and the relation called distance between pairs of points.

(2) The complete tabulation of distances between pairs of points may be arranged as follows:

(a) the points $P$ correspond to real number triples $(x, y, z)$;

(b) the squared distance between $P_{1}$ and $P_{2}$ is

$$
\left(x_{2}-x_{1}\right)^{2}+\left(y_{2}-y_{1}\right)^{2}+\left(z_{2}-z_{1}\right)^{2} .
$$

All of geometry follows very readily from these agreements. To begin with, the line segment $P_{1} P_{2}$ may be defined as the set of points $P$ such that the sum of the distance $P_{1} P$ and the distance $P P_{2}$ is the distance $P_{1} P_{2}$. The definition of a line segment makes the definition of a complete line possible of course, and then

2000 Mathematics Subject Classification. Primary 78A25, 70F07, 83C99.

Reprinted from Bull. Amer. Math. Soc. 33 (1927), 165-181.

Presidential Address, delivered before a joint meeting of this Society and Section A of the American Association for the Advancement of Science, December 30, 1926. This paper was awarded the Prize of the American Association for the Advancement of Science, for the 1926 meeting. 
of a plane, while the corresponding simple algebra yields the analytic equations of lines and planes. Similarly, the perpendicularity of two lines $l_{1}$ and $l_{2}$ with the common point $P$ can be defined as the relation existing between $l_{1}$ and $l_{2}$ when the sum of the squares of the two distances from any point on either line to the common point is equal to the square of the distance between these two points themselves. In this way one may successively define line segment, line, plane, perpendicularity, rectangular coordinate systems, etc. The whole body of geometrical fact with corresponding analytic framework is thus easily deducible, and yet one may stop at the fundamental principles, without taking up beautiful but less vital geometrical studies. More generally, an entirely analogous direct development of general differential geometry of $n$ dimensions, based on the well known differential formula for the squared element of distance, can be made. Indeed much of specialized geometry as well as of analysis is likely to give way some day, for as my predecessor, Professor Veblin, said in his address: "The main current of mathematics will flow by carrying with it only the more important facts." One thing is plain, however: it will remain the first duty of the mathematician to develop interesting and beautiful theories of all types, without being much concerned with the question of ultimate importance.

The theories of relativity of Einstein have made us appreciate that geometry arises directly out of the comparison of rigid bodies, and that its meaning becomes more and more precise as uniformity of pressure and temperature of the bodies, freedom from gravitational, rotational and other stresses are secured; but such precision is limited, because of the atomic structure of matter. In its origin the geometric concept of space is always to be associated with that of a corresponding body of reference.

\section{Classical dynamics}

Classical dynamics arises in the attempt to use euclidean space and absolute time as the means for expressing the laws of nature. Natural laws are then interpreted as describing the interaction of various particles, rigid and elastic bodies in a selected space of reference.

There lie at the very basis of this attempt to make space the container of matter, certain fundamental difficulties, to some of which I wish to direct your attention.

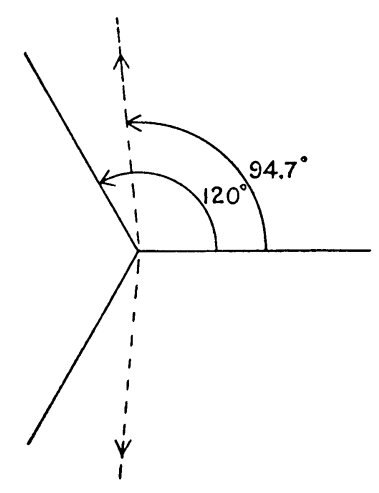

Figure 1 


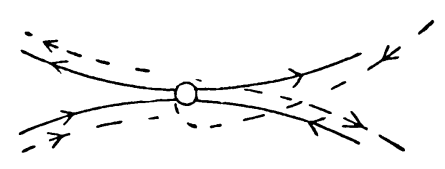

FIGURE 2

The simplest illustration of them arises in dealing with a collection of "equal rigid elastic spheres," which move in straight lines with uniform velocity except in so far as they may collide. The spheres will be taken to be non-rotating and smooth. This is the model on which the statistical treatment of the properties of a perfect gas is often based. When only two spheres collide, the assumed laws of contact action determine uniquely their directions and velocities under collision; but when more than two spheres collide, the situation is entirely different.

Suppose that three equal spheres approach a point with equal velocities, the lines of motion being $120^{\circ}$ apart and in the same plane. If all three spheres collide at the same instant, considerations of symmetry alone demand that the spheres must rebound back along the same lines with a velocity equal to that of approach. But it is easily verified that if two of them collide ever so little before they collide with the third, the resultant motion will be decidedly different in character, as indicated by the dotted lines of the figure. Such a result seems to contradict the fundamental physical requirement of continuity. In fact the laws of action suffice to determine the behavior of two spheres which collide, but as more and more complicated simultaneous collisions between three or more spheres are considered, it is not possible to infer their behavior by an argument based on continuity or even on symmetry. Hence these laws need to be supplemented by indefinitely many others of arbitrary type, if the mathematical theory is to be determinate.

The situation is similar with $n$ mass particles, attracting one another according to the Newtonian law. The laws of motion are then incorporated in $3 n$ differential equations of the second order. In the case of only three particles, if certain three area integral constants are not all zero, Sundman ${ }^{1}$ has shown that triple collision is impossible, and that the differential equations themselves suffice to define the motion after double collision; in fact, at double collision the bodies approach each other in a certain direction and may be taken to recede with nearly the same velocity in the opposite direction after collision; this is proper because for nearly a double collision the behavior is almost the same, as indicated by the dotted lines in the figure below. Hence considerations of continuity determine the behavior of collision.

But, if there are further particles, three particles may happen to collide simultaneously, and then there arises the same kind of indeterminateness as in the case when three rigid elastic spheres collide. Hence, here too, other supplementary conditions are required in addition to those furnished by the differential equations.

Finally, the concept of elastic bodies so fundamental in classical dynamics presents even more formidable logical objections. The whole of that theory is based on the notion of continuously distributed matter subject to certain strains and stresses obeying Hooke's law. It is a characteristic feature of the isotropic elastic body that the effects of any disturbance are propagated at a definite velocity, namely, the disturbance velocity. Bearing this property in mind, let us ask what happens when

\footnotetext{
${ }^{1}$ Acta Mathematica, 1912.
} 
two equal elastic spheres under no pressure approach along their line of centers with equal velocities which exceed the disturbance velocity. The parts of the spheres approaching the plane of collision have no possibility of reacting to the disturbance of collision since that plane is approaching the center of either sphere at a velocity greater than the disturbance velocity. On the other hand, the parts of the spheres which collide at the plane cannot rebound without interpretation. Thus it appears as if the spheres are converted into a kind of lamina of infinite density moving radially outward in the plane of symmetry. But this yields a total change of state, which the theory of elasticity does not contemplate.

Another but lesser stumbling block is contained in the following situation: Consider two equal elastic hemispheres at rest with the two circular boundaries in contact and compare these with a single sphere of the same size and also at rest. The differential equations, initial densities and pressures are identical and yet the reactions under the same force may be different, for in the first case the two hemispheres can be separated.

These illustrations show that the classical theory of particles, rigid and elastic bodies, needs to be supplemented by a variety of conditions, some of which do not appear to have been formulated, and that such formulation will of necessity be artificial in character.

There are other facts to be considered also. Hertz pointed out the peculiarities of such cases as an elastic sphere resting on a plane surface. Furthermore, if elastic spheres collide successively with one another, the internal kinetic energy will increase, while the relative velocities of the spheres becomes smaller and smaller. Thus a collection of small elastic spheres does not really furnish a model for a perfect gas. But these are questions of complication rather than of principle.

The central difficulty of indeterminateness disappears in the case of a single elastic body, but then the situation is no longer analogous to the case of a set of particles in empty space, which formed our starting point. The question now confronts us: Is it possible to conceive of simple laws of motion for systems of particles and for continuous bodies in empty space which will be unified and determinate?

To secure such a system of particles, it suffices to subject the particles to forces acting directly between them and of the form

$$
k m M\left(\frac{1}{r^{2}}-\frac{a}{r^{3}}\right),
$$

where $m$ and $M$ are the masses of the two particles, and $r$ is the distance between them; in other words, in addition to the ordinary Newtonian force of attraction there is a repulsive force inversely proportional to the cube of the distance. Since the potential energy of the system then increases indefinitely when any two particles approach collision, it follows that collision can never take place. Furthermore, if there are dissipative forces, there will be obviously a position of stable equilibrium for such a set of particles.

In order to deal with a continuous distribution of matter, we need merely to set up an appropriate potential energy function

$$
U=k \int\left(\frac{1}{r}-\frac{1}{2 r^{2}}\right) d m,
$$

where $d m$ is the element of mass and the integration is taken over space. This means that the law of force for the continuous distribution is the same as that for 
the system of particles just considered; the acceleration in any direction of a point of the body is given by the corresponding gradient of the potential function $U$. It is obvious that such a body cannot contract indefinitely since then its potential energy would exceed the total initial energy; nor can it expand indefinitely unless sufficient kinetic energy is available. Moreover, there will clearly be a single spherical state of equilibrium in case dissipative forces are present.

This type of body is instructive in another way. It illustrates how necessary it is to take the differential equations of motion as fundamental rather than to rely upon what appear to be simple physical intuitions. For, consider what happens if two such bodies collide. If we let ordinary intuition speak, we might declare that they will either rebound from one another or continue indefinitely in contact. But the nature of the differential equations of motion excludes both of these possibilities. In fact, when the bodies first touch, the points of contact have differing velocities, whereas the accelerations will be the same according to the formula. Consequently, the formula shows that they must interpenetrate rather than remain in contact or rebound from one another. Evidently such a fluid is entirely different in character from the elastic body under pressure, but it has at least the theoretical advantage of being free from the fundamental defect of indeterminateness. As perhaps of some interest, it may be remarked that two colliding bodies of this description will in general separate after a transitional period of interpenetration.

The chief mathematical instruments used by the physicists in dealing with space, time and matter according to the conception of classical physics have been the Lagrangian and Hamiltonian equations. I wish to digress somewhat from my main theme in order to make a remark concerning the nature and significance of these equations.

A dynamical system representable approximately by means of a set of "mass particles" subject to "rigid constraints" and "conservative forces" of gravitational or elastic character, has its potential energy given by a function of $n$ spatial coordinates, while its kinetic energy is expressed in terms of these coordinates and their velocities in the usual way. It is readily demonstrated that the equations of motion may be written in either of the above mentioned forms. The mathematician has thus been led to ask: What are the characteristics of equations of these types? In the particular case of the solar system, it was demonstrated by Laplace and Poisson that there would be partial stability. Poincaré proved that it was a general characteristic of equations of this type to possess complete formal stability. This means that the disturbances from periodic motion are essentially periodic in type and representable to all orders by means of trigonometric series with a certain degree of approximation.

I have recently succeeded in establishing a kind of converse result: If a dynamical system is such that its state is determined by $2 n$ coordinates, and if the perturbations from a periodic motion can be thus represented by trigonometric series, then the equations may be given Hamiltonian form. ${ }^{2}$ Thus perhaps the only significance of the Hamiltonian form of equations in classical dynamics is to insure automatically that periodic motions are completely stable. A more general Pfaffian type of equation seems better adapted to be useful to the physicist, for it possesses greater

\footnotetext{
${ }^{2}$ Comptes Rendus, September 20, 1926.
} 
ease and flexibility of transformation of the variables. I believe that the whole apparatus of transformation which is used in connection with the Hamiltonian equations has been overrated.

\section{The electromagnetic theory}

The equations of Maxwell, giving the interplay between the electric and magnetic forces in space, have never been modified, although it has been shown that these admit of very elegant formulation when presented in the Maxwell-Lorentz form. The space-time background corresponding to this form is that of the special theory of relativity, in which space and time are taken relative to some reference body and in which the velocity of light appears as a characteristic limiting disturbance velocity.

Fundamental in this theory is the notion of the minute test charge of electricity in the field, which is always supposed to be associated with ordinary matter. It is the acceleration of this electrified mass particle which measures the electric and magnetic forces in the field. In recent years statements have been made by physicists conjecturing that all mass is electromagnetic in origin. Now I have never been able to understand such a point of view, for in that case the ultimate particle of electricity would be "free" and would constantly move in directions determined by the electric and magnetic forces with a velocity equal to that of light.

A first question which arises is this: Shall the forces arising from the charge of the particle itself be considered as forming part of the total force? If we admit that this can be possible, we are confronted with the difficulty that the forces acting on the point particle will be infinite; but if we consider the resultant electromagnetic forces acting on a hypothetical small electrified sphere with the electrified particle as its center, the limiting forces can be determined. However, for a system of such particles there will be an indefinite radiation outward of electromagnetic energy as oppositely electrified particles fall into one another, while those similarly electrified tend to separate indefinitely under the mutual forces of repulsion. Evidently such a system of electrified particles is of little physical interest.

The use of an elastic fluid as the carrier of electricity seems at first sight to offer greater prospects of success. Not long ago I attempted to develop a theory based upon an adiabatic fluid having the property that the tension increased as the square of the density of the electrical charge. This tension was introduced in order to offset the electrical forces of repulsion within the proton and electron. It turned out that there was a unique sphere of equilibrium, of definite radius but of unstable type. This sphere contracted under slight displacement, and I tried to develop some of the properties of what I thought might be the final stable form. ${ }^{3}$

Further examination of the problem has made it appear impossible to secure stability of the kind desired, no matter what type of relation between pressure and density is assumed to hold. For, if we imagine the bits of the fluid to be widely distributed but under the same pressures and densities as at the outset, it is clear that the elastic potential energy is the same as before, while the electromagnetic energy of the field has been diminished. From this fact and some more purely mathematical considerations, it follows that there will always be a tendency toward breaking up of the fluid into smaller and smaller nuclei so that no final stable condition is possible.

\footnotetext{
${ }^{3}$ The Origin, Nature and Influence of Relativity, New York, the Macmillan Company, 1925. Chapters 6 and 7.
} 
But, aside from this instability which arises from the fact that electricity of one sign exerts strong forces of repulsion upon itself, there is another difficulty which arises even in the consideration of neutral matter not carrying any electrical charge. In fact, we have two types of disturbance velocities: firstly, that of light, and secondly, that of the elastic wave of the fluid. Now if the velocity of the latter wave is less than that of light, we can imagine two bodies approaching each other from opposite directions with velocities greater than the disturbance velocity but less than that of light. In this case the same paradox would arise as in the analogous classical case considered above.

Therefore the only possible elastic fluid would seem to be one with a disturbance velocity equal to that of light at all densities. The fluid of this type I shall term the "perfect fluid," by analogy with the ordinary perfect gas. The pressure in such a fluid is readily determined to be one half the density in absolute units, and so enormously great. Evidently the perfect fluid would tend to expand indefinitely with velocities approaching that of light. Of course such a perfect fluid would afford a much more unstable carrier of electricity than the one which I had proposed (loc. cit.). It should be noted in passing that because of the relativistic character of this perfect fluid, the mass of a small part of it is not invariable but changes as the square of the density of the attached charge.

Consequently, attempts to make use of an elastic fluid as the carrier of electricity seem to fail, and we are led to inquire whether it is not in the nature of the case that the elementary bodies such as the protons and electrons must be allowed to have some sort of autonomous existence in the same sense that empty space has. I shall make a suggestion of this sort in the "atomic potentials" used below. For the moment, however, I wish to emphasize the difficulty in maintaining the point of view of the "field" consistently. Consider a stretched elastic string which is vibrating longitudinally. No matter how the linear density or elastic coefficient varies, any local disturbance is propagated in both directions along the string with the disturbance velocity. Consequently the particle cannot be treated as a moving local disturbance. However, we might compare the particle to a bead on a string and moving with a velocity of its own, whose motion is affected by and affects that of the string. The bead would not appear then as a part of the string but would have autonomous existence. Here the strings correspond to the "field" and the bead to the body.

The idea of atomic potentials may be explained as follows. It is well known that the kinetic and elastic energy of an adiabatic fluid at low velocities can be defined just as in classical dynamics, in such wise that the principle of conservation of energy holds. Let us suppose in addition that there is an individual atomic potential energy of positive volume density, $\psi$, where $\psi$ has a value fixed for all time for each point of the fluid. This leads to a supplementary body force, proportional to the gradient of $\psi$ in space. In this way indefinite expansion of the corresponding proton or electron is prevented, for it would involve an indefinite increase in the atomic potential energy.

At first sight this seems to insure a stable spherical form of equilibrium. However, further consideration of small motions near the equilibrium state shows that the radial forces are so small as to make the nucleus amorphous under radial displacement, with a tendency to spread over space in wisp like form.

But now suppose that the protons are made of very small parts of the fluid with charge $+e$, while the electrons are made of parts of the perfect fluid with the charge 
$-e$, and with suitable atomic potentials. Let us suppose furthermore that such an electron can be penetrated freely by the proton.

Under these circumstances there will be a stable spherical form of equilibrium in which the proton coincides with the electron; for the tendency towards amorphous shape of the electrons and protons will be destroyed by the attractive forces between them.

The fact ought not to pass unnoted that all of the forces due to the atomic potential will have a resultant zero if the density of potential $\psi$ is constant at the boundary. Hence the electron as well as the proton will respond to external forces in the required way.

Here perhaps is a kind of two substance theory of matter and electricity which will be found to meet the fundamental mathematical requirements of determinateness and stability.

\section{General Relativity}

The essential logical structure of the gravitational theory of relativity of Einstein is extremely simple. Let us suppose that the universe of events is four-dimensional. Imagine a very slight disturbance of the existing physical condition to be made at an event. This disturbance will spread and modify physical phenomena in a certain region of space-time, in accordance with the principle of local causation. This region is analogous to a three-dimensional cone with vertex at the given event. Now the simplest mathematical form of definition for such a cone is that obtained from the vanishing of a differential quadratic form $d s^{2}$. Hence it seems probable that such a form $d s^{2}$ will lie at the base of any physical theory; in consequence, the symbolic language of tensor analysis becomes available. If we assume also that a mass particle moves in the space-time of general relativity according to the same underlying law as in the special theory of relativity (principle of equivalence) and if we determine the coefficients of $d s^{2}$ in such wise as to be as nearly as possible the same in character as the coefficients in the special theory, they become to a large degree determined.

Now it seems obvious that the space-time in the vicinity of the sun possesses spherical symmetry, and the law of motion obtained on this assumption is essentially that of Newton. In this way the fundamental experimental tests of Einstein are obtained.

Thus the general theory of relativity appears as a theory of empty space and throws no light upon the structure of matter. It is only the spherical symmetry of the space-time about the sun which allows us to come to a conclusion.

The logical situation is analogous to that involved in the following example. Suppose that a uniform square plate is maintained with two opposite sides at temperature $0^{\circ} \mathrm{C}$, and the other two sides at temperature $100^{\circ} \mathrm{C}$. I say that it is possible to prove that the temperature along the diagonals is exactly $50^{\circ} \mathrm{C}$, without making any other assumption than that states of temperature are additive. In fact, imagine the square rotated about one diagonal. In the new position the sides at temperature $0^{\circ}$ take the position of the sides previously at temperature $100^{\circ}$, and vice versa. By addition of the first and second states, a state is obtained at temperature $100^{\circ} \mathrm{C}$ around the four sides, and therefore $100^{\circ} \mathrm{C}$ within. But evidently along the invariant diagonal the temperatures of the two states are identical. In consequence the temperature along this diagonal, and similarly along the other, must be $50^{\circ} \mathrm{C}$. A like treatment of a rectangular plate would not be possible, because of the lesser symmetry. 
In attempting to extend the general theory of relativity to space occupied by matter, Einstein was led to "field equations"

$$
R_{i j}-\frac{1}{2} R g_{i j}=X T_{i j}, \quad(X=-8 \pi),
$$

where $T_{i j}$ is the "energy tensor" of matter and electricity. These equations are supposed to be supplemented by various "constitutive equations" such as the MaxwellLorentz equations. But it should be noted that there are in reality two types of energy tensors, namely, one for empty space and another for the interior of matter; for instance, it is only within matter that the velocity tensor is defined. Thus these are not really field equations as they stand.

The space-time framework of general relativity is adapted to our concept of atomic potential; for this purpose we define $T_{i j}$ as consisting of the usual elastic and electromagnetic energy tensor of an atomic potential term $\pi g_{i j}$. The mathematical fact that the divergence of the energy tensor vanishes will yield the observed law of motion of the protons and electrons.

\section{Atomic theory}

It is evident today that all earlier ideas in physics have been statistical in character, and that the fundamental laws are those of the atomic domain. The kinetic theory of heat illustrates this method of explanation of physical fact in the simplest possible way. Naturally the attention of physicists everywhere is fixed upon the discovery of the laws of the atom. The main theoretical attack is based upon electrical phenomena in rarefied gases and the properties of spectra.

If we grant the four-dimensional nature of space-time, it is natural to assume to argue by analogy about the atom's domain. This argument of continuity seems to make it imperative that the atom is an oscillating electromagnetic system, which radiates or absorbs electromagnetic energy.

Now the central facts about the atomic oscillator are essentially two: first, it acts like a combination of simple resonators of perfectly definite frequencies, such as those given by the Balmer formula in the case of the hydrogen atom; and secondly, these frequencies are excited only by means of certain quanta of energy (Planck's hypothesis).

Now in my opinion there need be no essential difficulty in accounting for this second fact. Imagine a pendulum to swing in a viscous medium whose viscosity diminishes rapidly as the distance from the position of equilibrium increases. With less than a certain initial velocity, the pendulum would swing back slowly towards its position of equilibrium, never passing it. On the other hand, if the initial velocity is sufficient to carry the pendulum beyond the zone of considerable viscosity, it will oscillate back and forth, traversing the viscous region in damped harmonic motion. Consequently we may conceive the so-called "energy levels" as defining the amount of energy necessary to carry the oscillators so far from equilibrium that they will move back and forth past the position of equilibrium. Thus the first and most fundamental task appears to be to find an oscillator possessing the desired frequencies. Afterwards one may investigate in detail the rate of electromagnetic radiation, which will correspond to viscosity.

It seems premature to abandon the attempt to explain the facts upon some such basis. Computations of the frequencies should be made for some simple conjectural theories of matter and electricity which meet the elementary mathematical demands of determinateness and stability. As far as I know this has not been done for a single 
case. The tendency of the quantum theory as developed by N. Bohr, Heisenberg, Born and others, has been altogether in the direction of obtaining a discrete theory of atomic structure. No doubt the discovery of a consistent theory of this kind would be of the utmost interest for mathematics as well as physics. But from my limited mathematical point of view, I can discern no kernel of thought in their work which tends toward the construction of a logically satisfactory discrete model, although this work is obviously of the highest value for physics.

Very recently Schrödinger ${ }^{4}$ has obtained a highly suggestive "wave equation," so that spectroscopic frequencies are treated by him as somewhat analogous to the frequencies of vibration of an electrodynamic system. He determines a sequence of frequencies and conjectures that the Balmer formula, which is obtained by taking the difference of two such frequencies, may be a difference frequency in the usual physical sense.

It will be of interest to compare his wave equation with that for the small oscillations of the fluid proton and electron in the theory outlined above. Now we observe that in this theory there figures a "substance coefficient" $\phi$ whose square gives the ratio of the density of matter to the square of the electrical density at any point; there is also the "atomic potential" $\psi$. Thus there are two functions $\phi$ and $\psi$ which may be given arbitrarily at the outset, and which need to be specified before definite conclusions can be made. I have not as yet had the opportunity of determining the boundary conditions and the actual frequencies. ${ }^{5}$ The three wave equations are of the same general type as the Schrödinger equation.

There is another remark about atomic dynamics that seems to me of importance. It is usual to approximate to an atomic problem by an ordinary dynamical problem such as the two-body problem, and the equations are then of Hamiltonian form. It is well known that any state of such a system tends to recur. Thus in general the motion will not be limited to certain periodic motions as demanded, or at least suggested, by the quantum theory. But, for a non-Hamiltonian system I have shown that there will exist a restricted set of "central motions" near which any motion will in general be found. ${ }^{6}$ Consequently if the central motions are periodic, we may expect a corresponding set of sharp frequencies. Thus an approximating set of differential equations may yield quantum orbits without any quantum conditions, provided that the equations are not of Hamiltonian type.

If I have convinced you of the necessity for a careful critique of the logical questions inherent in the laws ordinarily given for matter and electricity, I shall feel very well satisfied. The mathematician can be of great service in analyzing these basic ideas, in forming physical models of as simple type as possible which meet the most pressing physical requirements, and in making the necessary calculations of oscillation frequencies, etc. The possibilities of such models based upon an underlying four-dimensional space-time continuum have by no means been exhausted.

HARVARD UNIVERSITY

\footnotetext{
${ }^{4}$ Annalen der Physik, 1926.

${ }^{5}$ Further development of the theory outlined above will appear shortly in the Proceedings of the National Academy. The theory leads to a formula of the Balmer type for the frequencies.

${ }^{6}$ Göttinger Nachrichten, 1926.
} 